Objectives: The aim of our work was to investigate the factors influencing anxiety and depression in RA.

Methods: This was a cross-sectional study of 49 RA patients. We used the Hospital Anxiety and Depression scale (HAD) to assess anxiety disorders.

Results: We included 24 women and 5 men with an average age of 54.1 years. Sixty-nine percent of patients were unemployed and $81 \%$ had health insurance coverage. RA had been evolving for an average of $11.43 \pm 7.32$ years and the mean time to diagnosis was 2.35 years. It was erosive in $93.8 \%$ of cases. An atlanto-axial dislocation was found in $4.1 \%$ of cases and coxitis in $8.2 \%$ of cases. Eighty-three percent of patients were on cs-DMARDs and $14.2 \%$ were on biotherapy. Most patients had low active disease (53\%) with a mean DAS28CRP score of $2.74 \pm 0.81$. Twelve percent of patients had a probable anxiety and $18 \%$ had a probable depression.

A long delay in diagnosis was associated with a higher risk of anxiety disorders. Working patients had a lower risk of depression while patients from rural areas had a higher risk. The risk of anxiety and depression disorders was associated with increased VAS pain, EGP, and DAS28. In the multivariate study, EGP was the independent risk factor for the development of anxiety and depression disorders. Conclusion: Anxiety and depression disorders are a frequent yet underestimated consequence in RA. Appropriate care in psychiatry is required as soon as the diagnosis is announced.

Disclosure of Interests: None declared

DOI: 10.1136/annrheumdis-2021-eular.2066

\begin{tabular}{|l|l}
\hline AB0124 & STRUCTURAL DAMAGE IN THE FOOT IN \\
& RHEUMATOID ARTHRITIS: DON'T MISS THE OTHER \\
& METATARSOPHALANGEAL JOINTS
\end{tabular}

M. Yasmine ${ }^{1}$, H. Ferjani ${ }^{1}$, D. Kaffel ${ }^{1}$, K. Maatallah ${ }^{1}$, S. Rahmouni ${ }^{1}$, W. Hamdi ${ }^{1}$ ${ }^{1}$ Kassab Institute of Orthopedics, Rheumatology, Tunis, Tunisia

Background: The foot involvement in rheumatoid arthritis (RA) affects the functionality and the quality of life in patients. Despite this, the clinicians do not give enough care to the foot in RA patients, especially if asymptomatic, resulting in joint damage, deformity, and disability. The distribution of erosions of the other MTP joints (excluding the 5th) has not previously been studied.

Objectives: This study aimed to investigate the distribution of erosions in MTP joints and their clinical implications.

Methods: We conducted a retrospective study including patients with RA according to the American college of rheumatology/ the European league against rheumatism classification criteria. Sociodemographic data, as well as disease activity related characteristics, were recorded.

Posterior-anterior radiographs of the hands and feet of each patient were assessed for erosions.

All patients were assessed by Ultrasonography (US) of the hands. US erosions were scored 0-3 according to Szkudlarek [1]. We divided patients into two groups (G1 without MTPs erosions and G2 with MTPs erosions).

Results: We enrolled forty-two females and eleven males in our studies. The mean age was 58.6 years \pm 12.7 [23-77], and the mean disease duration was 8.4 years [1-47]. Rheumatoid factor or cyclic citrullinated peptide antibodies (Anti-CCP) were positive in $62.3 \%$ of cases. The mean DAS28ESR score was $5.1 \pm 1.16$ [2.5-7.7]. Half of the patients had the active disease $(52.8 \%)$. Hand erosions evaluated with plain radiographs and the US were found in $43.1 \%$ and $50.9 \%$ of cases, respectively. The distribution of foot erosions (15.1\%) was at follows: 5th right MTP $(7.5 \%)$, the 5 th right IPP (2\%), the 1st left MTP (2\%), 3th left MTP $(3.8 \%)$, 4th left MTP $(5.7 \%)$ and the 5th left MTP $(9.4 \%)$. Erosions on MTPs with the exclusion of the 5th MTP were present in $9.4 \%$ of cases. The presence of MTPs erosion was more frequent in males $(p=0.01)$ but was not associated with age $(p=0.6)$ or disease duration $(p=0.2)$. Seropositivity was similar between the two groups $(p=0.06)$. Similarly, the inflammatory markers (ESR and CRP), as well as DAS28 ESR, did not differ between the two groups ( $p>0.05)$. MTPs erosion was not associated with the presence of hand erosions on a plain radiograph $(p=0.445)$. However, MTPs erosion was significantly more frequent in patients with less erosive hands-on US $(p=0.034)$.

Conclusion: Our study showed that screening of other MTPs (excluding the 5th) is mandatory in RA diagnosis. Interestingly, in our result, MTPs erosion is more frequent in males with less erosive hands.

Disclosure of Interests: None declared

DOI: 10.1136/annrheumdis-2021-eular.2227

\section{AB0125 1 CLINICAL CHARACTERISTICS OF RHEUMATOID ARTHRITIS PATIENTS WITH IGG4-RELATED SYNOVITIS}

L. F. Chen ${ }^{1}$, X. Zhang ${ }^{1}$, C. Chen ${ }^{1}$, J. D. Ma ${ }^{1}$, Y. Mo ${ }^{1}$, J. Lin ${ }^{1}$, Y. Y. Zou ${ }^{1}$, D. H. Zheng ${ }^{1}$, L. Dai ${ }^{1}$. 'Sun Yat-Sen Memorial Hospital, Sun Yat-Sen University, Rheumatology, Guangzhou, China

Background: Elevated serum IgG4 (slgG4) and IgG4+ plasma cell tissue infiltration are outstanding features of IgG4-related disease (IgG4-RD). However, elevated
IgG4 is not specific for IgG4-RD. Our previous study reported elevated slgG4 in 46\% of rheumatoid arthritis (RA) patients (Mediators Inflamm 2014). Whether synovium from RA patients show similar characteristics of IgG4-RD and how about the clinical characteristics of RA patients with IgG4-related synovitis have not been reported yet. Objectives: To explore the serum and synovial IgG4 level and their correlation with disease indicators in RA.

Methods: Active RA patients who underwent needle synovial biopsy with qualified synovium tissue were recruited. Demographic and clinical data were collected simultaneously. Synovium tissue were stained with H\&E for Krenn synovitis score and immunohistochemistry for positive cell densities of CD20, CD38, IgG and IgG4. Serum IgG4 level was detected by immunonephelometry.

Results: Among 96 RA patients recruited, 74 (77.1\%) were female, the median age was 55.0 (46.0 61.0) years, disease duration was $42.0(12.0 \sim 120.0)$ months and SDAI was $31.2(22.1 \sim 42.8)$

The median slgG4 was 1.38 (0.86 2.42) $\mathrm{g} / \mathrm{L}$ and $49(51.0 \%)$ patients had elevated slgG4. Compared with those with normal slgG4, RA patients with elevated slgG4 had significantly higher levels of PrGA [7 (5 8) vs. 6 (4 7)], ESR [90 (64 116) mm/h vs. $61(38 \sim 75) \mathrm{mm} / \mathrm{h}]$, CRP $[46.20(17.20 \sim 74.20) \mathrm{mg} / \mathrm{L}$ vs. $18.90(9.46 \sim 49.20)$ $\mathrm{mg} / \mathrm{L}]$, DAS28-ESR $[6.3(5.6 \sim 7.4)$ vs. 5.7 (4.7 6.4)], SDAl [34.2 (25.3 48.8) vs 27.8 (18.9 35.9)] and HAQ-DI [1.70 (0.61 2.28) vs. $0.88(0.40 \sim 1.75)$, all $P<0.05]$ Meanwhile, they also showed significantly higher synovial counts of CD38+ plasma cells [1240(559 2290) $/ \mathrm{mm}^{2}$ vs. 1020(354 1777) $\left./ \mathrm{mm}^{2}\right]$, IgG4+ plasma cells [106 (39 249) $/ \mathrm{mm}^{2}$ vs. $68(3 \sim 123) / \mathrm{mm}^{2}$ ], and higher ratio of IgG4+/lgG+ plasma cells [26.3 (15.5 38.0) \% vs. $15.2(0.9 \sim 24.7) \%$, all $P<0.05$ ]

The median IgG4+ plasma cells count was $83(10 \sim 192) / \mathrm{mm}^{2}$ and median ratio of IgG4+/lgG+ plasma cells was $19.1(8.4 \sim 31.5) \%$. Both of them correlated positively with ESR, CRP and slgG4 $(r=0.216 \sim 0.394$, all $P<0.05)$. There were 46 $(47.9 \%)$ patients with IgG4+ plasma cells $>10 / \mathrm{HPF}$, who had significant higher ESR [86 (50 109) mm/h vs. 65 (40 84) mm/h] and CRP [43.35 (16.93 77.85) $\mathrm{mg} / \mathrm{L}$ vs. $26.15(9.54 \sim 52.53) \mathrm{mg} / \mathrm{L}$, both $P<0.05]$ than those with IgG4+ plasma cells $\leq 10 / \mathrm{HPF}$. There were $13(13.5 \%)$ patients with the ratio of $\operatorname{lgG} 4+/ \lg \mathrm{H}_{+}$ plasma cells $>40 \%$, and $11(11.5 \%)$ patients with both IgG4+ plasma cells $>10 /$ HPF and IgG4+/lgG+ plasma cells ratio $>40 \%$ (IgG4-related synovitis). RA patients with IgG4-related synovitis had significant higher ESR than the others [106 (53 125) mm/h vs. 69 (41 91) $\mathrm{mm} / \mathrm{h}, P<0.05$ ]

There were 10 (10.4\%) patients showing elevated slgG4 and IgG4-related synovitis. Four patients completed 1-year follow-up and all of them achieved remission at $6^{\text {th }}$ month (SDAl $\leq 3.3$, Figure 1). Only one patient had radiographic progression at $12^{\text {th }}$ month.
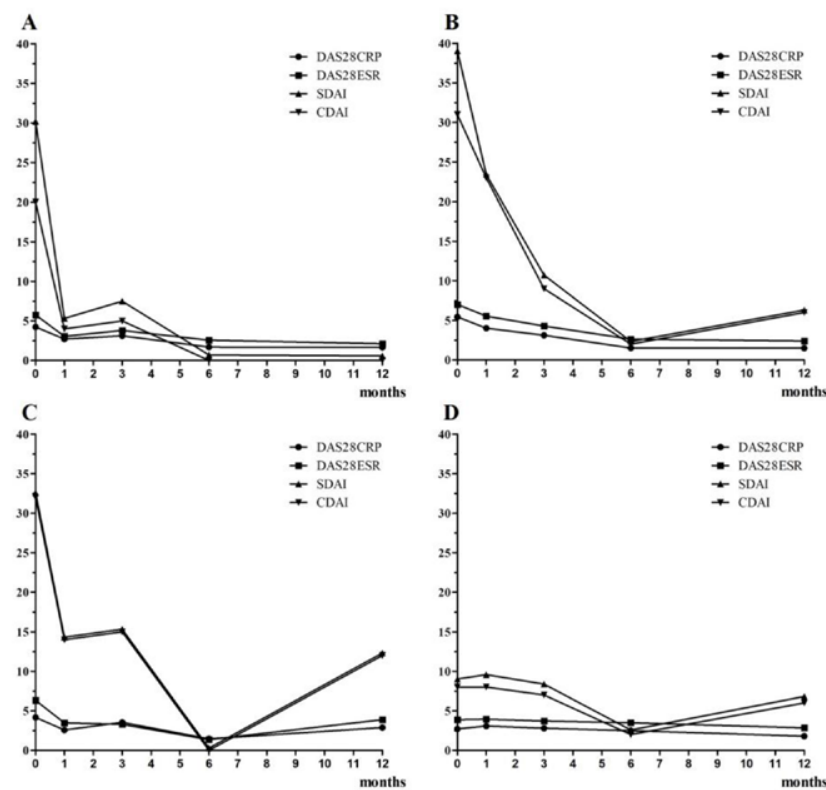

Figure 1. Dynamic disease activity of 4 RA patients with elevated slgG4 and IgG4-related synovitis during 1-year follow-up.

Conclusion: IgG4-related synovitis can be found in RA patients. Their clinical significance in disease characteristics and outcomes are worth further study. Acknowledgements: This work was supported by National Natural Science Foundation of China (no. 81971527, 81801606 and 81801605), Guangdong Natural Science Foundation (no. 2018A030313541 and 2018A030313690) Guangdong Medical Scientific Research Foundation (no. A2018062), Guangdong Basic and Applied Basic Research Foundation (no. 2019A1515011928 and 2020A1515110061), and Science and Technology Program of Guangzhou (no. 201904010088) 
Disclosure of Interests: None declared

DOI: 10.1136/annrheumdis-2021-eular.2245

\section{AB0126 METHOTREXATE MAINTENANCE THERAPY DURING THE TARGETED THERAPY ERA IN RHEUMATOID ARTHRITIS}

S. Abdellaoui ${ }^{1}$, S. Lefkir ${ }^{1} .{ }^{1}$ Issad Hassani Beni Messous Hospital, Rheumatology, Algiers, Algeria

Background: The persistence with methotrexate (MTX) at 1 year or 5 years in rheumatoid arthritis (RA) is very variable and intolerance remains the main cause of discontinuation of treatment. Changes in treatment with the strategy of adding "add-on" to a targeted therapy when the conventional DMARD becomes insufficient to optimally control the disease may decrease the maintenance MTX therapy rates, particularly for biologics with a Marketing Authorization as monotherapy. Objectives: The main objective of this study was to determine the rate of maintenance at 1 year of the combination therapy with different biologics, to compare the evaluation scores in patients receiving treatment as monotherapy (biological only) versus combination therapy with methotrexate and to analyze predictive factors for MTX maintenance therapy.

Methods: We performed a descriptive study of 56 patients with RA meeting the criteria of the ACR 2010. Statistical analysis SPSS.20 Software.

Results: These were 56 patients: 93\% women and 7\% men, mean age 46.74 years with an average duration of the disease of 14.74 years. $71.42 \%$ of patients were under corticosteroids taken for a period of 13 years with an average dose of $5.04 \mathrm{mg} /$ day. BMI was highin $23.80 \%$ of patients, $30.95 \%$ had at least one associated comorbidity. The RF was positive in $61.90 \%$ and ACPA positive in $78.57 \%$ of cases. $47.62 \%$ were under MTX taken for 6.55 years with a dose of $13.03 \mathrm{mg} /$ week on average: $68.75 \%$ by oral intake and $31.25 \%$ by subcutaneous intake with an average duration of setting before the switch of 8 years. 28 patients were on rituximab, 19 patients on tocilizumab and 9 patients on TNFi. 88.09\% did not havenot received from bDMARD before. The mean ESR was $36.57 \mathrm{~mm}$ $\mathrm{H} 1$ and CRP was $8.56 \mathrm{mg} / \mathrm{L}$. DAS28 at baseline was 6.81 and the current DAS 28 was 2.95 and the HAQ was 1.11 . The rate of MTX maintenance therapy at 1 year was $36.84 \%$, with rituximab, $64.28 \%$ with tocilizumab and $88 \%$ with TNFi. The comparison between the combination therapy with MTX and monotherapy groups showed a significant difference for the number of tender joints NTJ (3.5 vs 2.61), however, no significant difference concerning DAS28 value (2.98 vs 3.06), number of swollen joints NSJ (0.29 vs 0.22$)$ and HAQ (1.09 vs 1.15). Regarding predictive factors of MTX maintenance therapy: Significant correlation was found with a high DAS 28 at baseline, however no correlation concerning the positivity of ACPA, duration of the disease, NTJ, NSJ, ESR, HAQ and the high dose of MTX. Reduction in persistence has found a significant correlation with non-naive RA of $\geq 1$ bDMARD, disease activity score, type of biotherapy (antiTNF / rituximab vs Tocilizumab), young age, however no influence was found with a high $\mathrm{BMI}, \mathrm{HAQ}$, the presence of comorbidities or the withdrawal of corticosteroids. The increase in the persistence of combination therapy was associated with male gender and RF seropositivity only.

Conclusion: Drug persistence is an important aspect of treatment effectiveness. For rheumatologists, knowledge of the factors that predict whether to maintain the combination therapy with methotrexate, increase or reduce the persistence is of great interest when choosing a new treatment to initiate in patients with RA. REFERENCES:

[1] Lavielle.M and Dougados.M. Targeted therapies in rheumatoid arthritis:

Combination with conventional synthetic disease modifying antirheumatic drugs or monotherapy? Jone Bone Spine 2018;85:3-9.

Disclosure of Interests: None declared

DOI: 10.1136/annrheumdis-2021-eular.2346

\section{AB0127 1 COMBINATION THERAPY WITH METHOTREXATE AND QUALITY OF LIFE IN RHEUMATOID ARTHRITIS}

S. Abdellaoui ${ }^{1}$, S. Lefkir ${ }^{1} .{ }^{1}$ Issad Hassani Beni Messous Hospital, Rheumatology, Algiers, Algeria

Background: The level of quality of life in patients with rheumatoid arthritis (RA) is often overlooked. These patients suffer from an often-precarious quality of life resulting in pain, joint destruction and fatigue.
Objectives: The main objective of this study was to compare the level of quality of life in patients with RA receiving treatment with disease-modifying anti-rheumatic drugs (DMARDs): monotherapy (biological only) versus combination therapy with methotrexate.

Methods: We performed a descriptive study of 56 patients with RA meeting the criteria of the ACR 2010. The SPSS.20 software was used for statistical analyses. Results: These were 56 patients: 93\% women and 7\% men, mean age 46.74 years with an average duration of the disease of 14.74 years. 28 patients were on rituximab, 19 patients on tocilizumab and 9 patients on TNFi. The mean HAQ was $1.11 .52 .38 \%$ of patients were on biological alone as monotherapy. The Compliance Questionnaire Rheumatology score (CQR19) was 55.15 / 100, the RAID (Rheumatoid Arthritis Impact of Disease) score was 3.08 / 10, the SF36 of 56.01 / 100, the AIMS score of 2.10 / 10 for social activity, 2.44 / 10 for pain, 3.32 / 10 for depression and 4.06 for physical activity.

The comparison between the 2 groups (Combination therapy vs monotherapy) did not find any significant difference in terms of quality of life parameters: An SF-36 score $>55$ was found in $56 \%$ in patients on combination therapy vs. $44 \%$ on monotherapy, the RAID score was 3.02 vs. 3.12 , AIMS social activity 2.08 vs 2.13 , AIMS pain 2.33 vs 2.59 , AIMS activity physics of 4 vs 4.11

Conclusion: Our study did not demonstrate any superiority of the combination with methotrexate in improving quality of life. The use of biotherapy in patients with RA has been shown to be an important pharmacological strategy for the overall management of the disease.

\section{REFERENCES:}

[1] Lavielle.M and Dougados.M. Targeted therapies in rheumatoid arthritis: Combination with conventional synthetic disease modifying antirheumatic drugs or monotherapy? Jone Bone Spine 2018; 85:3-9.

Disclosure of Interests: None declared

DOI: 10.1136/annrheumdis-2021-eular.2453

\section{AB0128 1 CONSTRUCTION OF THE VETERANS AFFAIRS NATIONAL RHEUMATOID ARTHRITIS DATABASE (VANRAD)}

A. Joseph ${ }^{1,2}$, J. Yanagida ${ }^{2}$, X. Huang ${ }^{1,2}$, P. Ranganathan ${ }^{1,2}$, M. Laurie ${ }^{3}$, H. Xian ${ }^{2,4}$, S. Eisen ${ }^{1,2}$. ${ }^{1}$ Washington University School of Medicine, Rheumatology, Saint Louis, Missouri, United States of America; ${ }^{2}$ VA St. Louis Health Care System, Rheumatology, Saint Louis, Missouri, United States of America; ${ }^{3}$ Bristol Myers Squibb, Health Economics \& Outcomes Research, Lawrence Township, United States of America; ${ }^{4}$ Saint Louis University College for Public Health \& Social Justice, Epidemiology \& Biostatics, Saint Louis, Missouri, United States of America

Background: The Department of Veterans Affairs (DVA) provides comprehensive medical care at minimal or no cost to 9 million veterans annually through 170 medical centers and 1074 outpatient clinics across the United States. In 1999, the DVA established a national, fully integrated electronic health record (EHR), which now includes approximately 24 million veterans. However, few studies have used VA EHR data to examine the validity of diagnoses of rheumatoid arthritis (RA). Objectives: Develop a validated, national database (VANRAD) of patients with RA who received VA care since International Classification of Diseases, tenth revision (ICD-10) coding was introduced in 2015. The database will provide infrastructure for retrospective and prospective research to address the 'real-world' care of patients with RA.

Methods: Patients with the following criteria were identified from the VA EHR as of October 2, 2020: (a) $\geq 1$ ICD-10 diagnosis code of RA; (b) treatment with $\geq 1$ disease-modifying anti-rheumatic drug (DMARD); (c) $\geq 2$ VA rheumatology clinic visits; and $(d) \geq 1$ rheumatoid factor (RF) or anti-cyclic citrullinated peptide (aCCP) antibody test result. From this group, 553 EHRs were randomly selected for review. The 'gold standard' for the diagnosis of RA was the treating rheumatologist's diagnosis, documented in the EHR.

Results: 27,482 patients met eligibility criteria. Sociodemographic characteristics were: $85.6 \%$ male, mean age of 69.7 years $(y)(S D=10.9$ y; range $=21.9$ y to $100.5 \mathrm{y}), 76.4 \%$ white, $17.0 \%$ African American; and mean VA care duration 14.1 y $(S D=5.3 y$, range $=0.04$ y to $20.0 \mathrm{y})$.

For patients with $\geq 1 \mathrm{RF}$ or aCCP test, the positive predictive value (PPV) for RA ranged from $65.3 \%$ (aCCP-/RF-) to $95.8 \%$ (aCCP+/RF+); rheumatologists' likelihood of a 'possible' diagnosis was higher if the aCCP test result was negative or not available (Table 1). Excluding patients with a second rheumatologic diagnosis did not improve PPV results (data not shown). 